\title{
ENTEROPARASITOS EM AMOSTRAS DE Lactuca sativa EM UM MUNICÍPIO NO ESTADO DE MINAS GERAIS
}

Meiriane Sanches Colombo ${ }^{1}$ Renata Gregório Franco Moura²

Gabriela Lícia Santos Ferreira ${ }^{3}$

César Gómez Hernandéz ${ }^{4}$

Karine Rezende Oliveira

COLOMBO, M. S.; MOURA, R. G. F.; FERREIRA, G. L. S.; HERNANDÉZ, C. G.; OLIVEIRA, K. R. Enteroparasitos em amostras de Lactuca sativa em um município no estado de Minas Gerais. Arq. Ciênc. Vet. Zool. UNIPAR, Umuarama, v. 21, n. 1, p. 33-36, jan./mar. 2018.

RESUMO: Investigou-se a presença de formas evolutivas de enteroparasitos em alfaces (Lactuca sativa) vendidas em diferentes áreas de distribuição urbanas em Ituiutaba, Minas Gerais. Foram analisados 72 pés de alface, utilizando as técnicas de sedimentação espontânea e de Ritchie, com o intuito de encontrar cistos e ovos de enteroparasitos. Observouse a presença de enteroparasitos em 75\% das amostras oriundas de sacolões, sendo, Endolimax nana (90,7\%), ascarídeos (29,6\%), ancilostomídeos (18,5\%), Strongyloides sp. (16,7\%), Entamoeba histolytica/E. dispar (9,2\%) e Entamoeba coli $(3,7 \%)$. Das amostras advindas das feiras livres, 16,7\% foram positivas, sendo, E. nana (91,7\%), ancilostomídeos (33,3\%), ascarídeos (25\%), E. histolytica/E. dispar (16,7\%) e Strongyloides sp. (8,3\%). Quanto às alfaces da horta, 8,4\% mostraram-se positivas, sendo, E. nana (66,6\%), ascarídeos (50\%), Strongyloides sp. (16,7\%), E. histolyticalE. dispar (16,7\%) e Toxocara sp. (16,7\%). As alfaces comercializadas em diferentes estabelecimentos da área estudada apresentaram condições de higiene, manipulação e acondicionamento inadequados.

PALAVRAS-CHAVE: Enteroparasitoses. Hortaliças. Prevalência.

\section{ENTEROPARASITES FROM SAMPLES OF Lactuca sativa IN A CITY IN THE STATE OF MINAS GERAIS}

\begin{abstract}
The presence of enteroparasite forms in lettuce (Lactuca sativa) sold in different urban distribution areas in the city of Ituiutaba, Minas Gerais, was investigated. Seventy-two lettuce samples were analyzed, using spontaneous sedimentation and Ritchie techniques, searching for enteroparasite cysts and eggs. The presence of enteroparasites was observed in 75\% of the samples from supermarkets, being Endolimax nana (90.7\%), ascarids (29.6\%), hookworms (18.5\%), Strongyloides sp. (16.7\%), Entamoeba histolyticalE. dispar (9.2\%), and Entamoeba coli (3.7\%). On samples obtained from street markets, $16.7 \%$ presented positive results, being E. nana (91.7\%), hookworms (33.3\%), ascarids (25\%), E. histolytica/E. dispar (16.7\%), and Strongyloides sp. (8.3\%). As for the lettuce from the vegetable garden, $8.4 \%$ were positive, being, E. nana (66.6\%), ascarids (50\%), Strongyloides sp. (16.7\%), E. histolytica/E. dispar (16.7\%) and Toxocara sp. (16.7\%). Lettuce sold in different stores in the studied area presented inappropriate hygiene, handling and packaging conditions.
\end{abstract}

KEYWORDS: Enteroparasitoses. Greens. Prevalence.

\section{ENTEROPARÁSITOS EN MUESTRAS DE Lactuca sativa EN UN MUNICIPIO EN EL ESTADO DE MINAS GERAIS}

RESUMEN: Se investigó la presencia de formas evolutivas de enteroparásitos en lechuga (Lactuca sativa) vendidas en diferentes áreas de distribución urbana en Ituiutaba, Minas Gerais. Se analizaron 72 muestras de lechuga, utilizando las técnicas de sedimentación espontánea y de Ritchie, con el propósito de encontrar quistes y huevos de enteroparásitos. Se observó la presencia de enteroparásitos en $75 \%$ de las muestras procedentes en puntos de venta, siendo, Endolimax nana (90,7\%), ascárideos (29,6\%), ancilostomídeos (18,5\%), Strongyloides sp. (16,7\%), Entamoeba histolytica/E. dispar (9,2\%) y Entamoeba coli (3,7\%). De las muestras procedentes de las ferias libres, 16,7\% fueron positivas, siendo, E. nana (91,7\%), ancilostomídeos $(33,3 \%)$, ascarídeos $(25 \%)$, E. histolyticalE. dispar $(16,7 \%)$ y Strongyloides sp. (8,3\%). En cuanto a las lechugas de la huerta, 8,4\% fueron positivas, siendo, E. nana (66,6\%), ascarídeos (50\%), Strongyloides sp. (16,7\%), E. histolytica/E. dispar (16,7\%) y Toxocara sp. (16,7\%). Las lechugas comercializadas en diferentes establecimientos del área estudiada presentaron condiciones de higiene, manipulación y acondicionamiento inadecuados.

PALABRAS CLAVE: Enteroparásitos. Hortalizas. Prevalencia.

DOI: 10.25110 /arqvet.v21i1.2018.6624

${ }^{1}$ Bióloga. Especialização em Higiene e Alimentos. Rua 20, 1600, Tupã, CEP 38304402, Ituiutaba - MG. meirianesanches@hotmail.com ${ }^{2}$ Bióloga. Mestre em Medicina Tropical e Infectologia. Rua 20, 1600, Tupã, CEP 38304402, Ituiutaba - MG. renata gfm@hotmail.com ${ }_{3}^{3}$ Bióloga. Doutora em Imunologia e Parasitologia Aplicadas. Rua 20, 1600, Tupã, CEP 38304402, Ituiutaba - MG. gabriela@ufu.br ${ }^{4}$ Médico. Doutor em Medicina Tropical e Infectologia. Rua Frei Paulino, 30, Abadia, CEP 38025180, Uberaba - MG. cesar_cgh@hotmail.com ${ }_{5}^{5}$ Bióloga. Doutora em Medicina Tropical e Infectologia. Rua 20, 1600, Tupã, CEP 38304402, Ituiutaba - MG. karinerezende@ufu.br* 


\section{Introdução}

Frutas e hortaliças consumidas cruas e sem a adequada higienização constituem um importante meio de disseminação de cistos, ovos e larvas de enteroparasitos (PERES JUNIOR et al., 2012).

Alguns métodos de controle e higiene podem ser utilizados como meios profiláticos, como a utilização de filtros de água, instalação de fossas e rede de esgoto para evitar-se a contaminação do solo e da água para irrigação, além da manipulação mais cuidadosa das verduras, desde a colheita até sua preparação (COELHO et al., 2001; MOHAMED et al., 2016; BEKELE et al., 2017).

Considerando-se a importância clínica e epidemiológica das diferentes parasitoses intestinais, o presente estudo buscou verificar a presença de formas evolutivas de enteroparasitos em touceiras de alface (Lactuca sativa) obtidas em diferentes áreas de distribuição urbana e de fácil acesso à população do município de Ituiutaba - MG.

\section{Material e Métodos}

Participaram do estudo 12 estabelecimentos (nove sacolões, duas feiras livres e uma horta doméstica), sendo que, em cada local, foram adquiridas duas touceiras de alface, em dias distintos, no período de setembro de 2011 a maio de 2012. Seguindo o método de (COELHO et al., 2001), as alfaces foram desfolhadas, separadas em amostras contendo 100 gramas de cada hortaliça e mergulhadas em $200 \mathrm{~mL}$ de água destilada, os quais ficaram em repouso por 12 horas. Após este período, as folhas foram raspadas dentro desta água com o intuito de retirar todo o material de interesse. Em seguida, foram introduzidas em centrífuga manual e centrifugadas por dois minutos, para remoção total da água das hortaliças. A água resultante da lavagem/raspagem e da centrifugação manual foi depositada em cálices de vidro para sedimentação do material durante 24 horas.

Após este período, foram colhidos $2 \mathrm{~mL}$ do sedimento e colocados em tubos cônicos de $15 \mathrm{~mL}$, juntamente com $4 \mathrm{~mL}$ de solução fisiológica $0,9 \%$ e centrifugados por 10 minutos a $1500 \mathrm{rpm}$. Em seguida, foram pipetados $50 \mu \mathrm{l}$ do sedimento, corados com solução de lugol 5\% e examinado em microscópio óptico em objetivas de $10 \times$ e $40 \times$. As amostras foram analisadas também pelo método de Ritchie e $50 \mu \mathrm{l}$ do sedimento resultante foi pipetado em lâmina, corado com a solução de lugol 5\% e levado ao microscópio óptico para a identificação das formas parasitárias. Foram confeccionadas três lâminas por amostra de sedimento.

Para a análise das médias totais do número de cistos de protozoários, ovos e larvas de helmintos encontrados e avaliar a diferença entre os locais de coleta e a presença de parasito foi aplicado o teste de $C h i$-Quadrado $\left(\chi^{2}\right)$. A análise foi considerada estatisticamente significativa para os valores de $p<0,05$, utilizando o programa Statsoft Statistica 8.0.

\section{Resultados}

Ao todo, foram adquiridas 72 touceiras de Lactuca sativa nos 12 estabelecimentos e todas apresentaram alguma forma parasitária. Em 28 amostras $(38,9 \%)$ encontrou-se apenas uma espécie de parasito e, em 44 (61,1\%) amostras, observou-se mais de uma espécie de parasito. As formas parasitárias observadas foram cistos de Endolimax nana $(88,8 \%)$, ovos de ascarídeos (30,5\%), ovos de ancilostomídeos (19,4\%), larvas de Strongyloides stercoralis (15,3\%), cistos de Entamoeba (E.) histolytica/E. dispar (11,1\%), cistos de E. coli $(2,8 \%)$ e ovos de Toxocara sp (1,5\%).

Nos sacolões, foram colhidas 54 (75\%) amostras de alface e foram observados cistos de E. nana (90,8\%), ovos de ascarídeos (29,6\%), ovos de ancilostomídeos (18,5\%), larvas de $S$. stercoralis $(16,7 \%)$, cistos de E. histolyticalE. dispar $(9,2 \%)$ e cistos de E. coli $(3,7 \%)$. Nas feiras livres, foram colhidas $12(16,7 \%)$ amostras e observados cistos de E. nana $(91,7 \%)$, ovos de ancilostomídeos $(33,3 \%)$, ovos de ascarídeos (25\%), cistos de E. histolytica/E. dispar $(16,7 \%)$ e larvas de $S$. stercoralis $(8,3 \%)$. Na horta, foram colhidas seis $(8,4 \%)$ amostras de alface e observados cistos de E. nana (66,6\%), ovos de ascarídeos (50\%), larvas de S. stercoralis $(16,7 \%)$, cistos de E. histolytica/E. dispar (16,7\%) e ovos de Toxocara sp. $(16,7 \%)$.

Em todos os locais de coletas (sacolões, feiras livres e horta), houve diferença significativa quanto à porcentagem de cistos de $E$. nana encontrada em relação às outras espécies de parasitos $\left(\chi^{2}=0,046 ; \mathrm{p}<0,05\right)$. Não houve diferença estatística entre as taxas de parasito detectados nos locais de coleta: horta em relação ao sacolão $\left(\chi^{2}=0,0814\right)$, feira livre comparado ao sacolão $\left(\chi^{2}=0,9196\right)$ e feira livre em relação a horta $\left(\chi^{2}=0,1797\right)$.

\section{Discussão}

O consumo de frutas e verduras in natura proporciona benefícios à saúde, contudo, existem fatores que favorecem a ocorrência de doenças parasitárias por meio da ingestão desses alimentos cultivados em áreas contaminadas com dejetos fecais, irrigados com águas poluídas ou acondicionados em locais impróprios (SILVA et al., 2005; YUSOF et al., 2017).

Neste trabalho foi possível verificar que todas as amostras apresentaram alguma forma parasitária, assim como ocorreu no trabalho de Guimarães e colaboradores em 2003, que analisou 120 amostras de alfaces oriundas de supermercados, sacolões e feiras livres da cidade de Lavras, Minas Gerais, encontrando helmintos e protozoários em todas as suas amostras (GUIMARÃES et al., 2003). Tais resultados se justificam pelo fato de que hortaliças com folhas múltiplas, como alface e outras folhosas, possuem uma extensa área de contato, o que permite a fixação de formas parasitárias, além de estarem em contato com o solo por um maior tempo (BEKELE et al., 2017). A ocorrência de poliparasitismo nas amostras deste estudo reflete a possibilidade da contaminação multi-fecal dos locais visitados, o que pode levar a múltiplas infecções parasitárias em seres humanos.

Devido ao fato dos sacolões proporcionarem à população um acesso facilitado aos produtos hortícolas, pelo preço acessível, evidencia-se uma maior exposição das hortaliças a fatores que contribuem com a contaminação das mesmas e dos consumidores (SOARES; CANTOS, 2005). Além dos sacolões, existem as feiras livres, que são locais de fácil acesso às hortaliças e, que na maioria das vezes, é possível determinar a procedência da colheita e qualidade dos produtos, mas o transporte e acondicionamento dos mesmos 
de forma errônea, pode favorecer a contaminação por enteroparasitos (SOARES; CANTOS, 2005). As hortas também podem representar um risco de contaminação dos alimentos, já que vegetais que crescem em solos poluídos podem carrear enteroparasitos (ROBERTSON; GJERDE, 2001).

Foi encontrada uma alta prevalência de $E$. nana e $E$. coli nas amostras e, embora esses parasitos não sejam considerados patogênicos, é importante destacar que sua presença indica uma contaminação fecal do ambiente, pois o mesmo é um parasito intestinal do homem, o que pode sugerir falha na higienização ou manipulação das hortaliças (PAULA et al., 2003; ANSELMO et al., 2015). Também foi verificada a presença de cistos de E. histolytica/E. dispar nas amostras, assim como no estudo de Jung e colaboradores, em 2014, que encontraram uma alta taxa destes protozoários nas amostras estudadas, sendo este resultado preocupante por se tratar da espécie que causa a amebíase, representando um problema de saúde pública (ZUMAQUERO-RIOS et al., 2013; DUEDU et al., 2014; JUNG et al., 2014).

Neste estudo, foram detectados ovos de Toxocara sp. e ancilostomídeos, o que pode estar relacionado com a contaminação dos solos com ovos de parasitos, originários de cães ou gatos, presentes nas plantações ou devido ao comprometimento do sistema de irrigação (MOHAMED et al., 2016), sendo indicado que estes animais sejam vermifugados periodicamente para diminuir a possível contaminação do solo (GAWOR; BORECKA, 2017).

Foi detectada a presença de larvas de Strongyloides sp. nas amostras coletadas em todos os estabelecimentos visitados. As larvas encontradas foram do tipo rabditóide (não-infectante), porém, as mesmas podem ser liberadas por meio das fezes de um indivíduo já parasitado, e evoluir em meio externo para a forma infectante (filarióide) e para adultos de vida livre, gerando risco de infecção para os horticultores (TEFERA et al., 2014).

\section{Conclusão}

O estudo mostrou que todas as amostras de alface analisadas nos estabelecimentos apresentaram alta prevalência de enteroparasitos com potencial patogênico, ressaltando a importância da realização de novos estudos na área, pela ausência de pesquisas sobre a ocorrência de parasitos em amostras de legumes ou verduras em locais públicos no município. Ressalta-se a necessidade em se adotar medidas profiláticas como a melhoria da qualidade da água de irrigação, lavagem adequada das hortaliças antes do consumo, instalação de fossas sépticas e redes de esgoto e, principalmente, promoção de saúde, de forma a informar e educar a população, contribuindo com diminuição e propagação das enteroparasitoses.

\section{Referências}

ANSELMO, D. B.; WERLE, C. H.; HOFFMANN, F. L. Occurrence of antibiotic-resistant Escherichia coli and Staphylococcus aureus and of Entamoeba coli and Ascaris lumbricoides parasites in the school snacks. Revista do Instituto Adolfo Lutz, v. 74, p. 399-409, 2015.

BEKELE, F. et al. Parasitic contamination of raw vegetables and fruits collected from selected local markets in Arba Minch town, Southern Ethiopia. Infectious Diseases of Porvety, v. 6, p. 1-7, 2017.

COELHO, L. M. P. S. et al. Detection enteroparasites transmissible forms in water and raw vegetables consumed in pre-schools from Sorocaba, São Paulo State, Brazil.

Revista da Sociedade Brasileira de Medicina Tropical, v. 34, p. 479-482, 2001.

DUEDU, K. O. et al. A comparative survey of the prevalence of human parasites found in fresh vegetables sold in supermarkets and open-aired markets in Accra, Ghana. BMC Research Notes, v. 7, p. 1-6, 2014.

GAWOR, J.; BORECKA, A. Quantifying the risk of zoonotic geohelminth infections for rural household inhabitants in Central Poland. Annals of Agricultural and Environmental Medicine, v. 24, p. 44-48, 2017.

GUIMARÃES, A. M. et al. Frequency of intestinal parasites in samples of lettuce (Lactuca sativa) commercialized in Lavras, Minas Gerais State. Revista da Sociedade Brasileira de Medicina Tropical, v. 36, p. 621-623, 2003.

JUNG, G. J. et al. Parasitos em alface Lactuca Sativa (Asterales: Asteraceae) cultivadas em pequenas propriedades rurais dos municípios de Capinzal, Vargem Bonita e Lacerdópolis, Santa Catarina, Brasil. Unoesc \& Ciência, v. 5, p. 103-108, 2014.

MOHAMED, M. A. et al. Parasitic contamination of fresh vegetables sold at central markets in Khartoum state, Sudan. Annals of Clinical Microbiology and Antimicrobials, v. 15, p. 1-7, 2016.

PAULA, P. et al. Microbiological and parasitological contamination of lettuce (Lactuca sativa) from self service restaurants of Niterói city, RJ. Revista da Sociedade Brasileira de Medicina Tropical, v. 36, p. 535-537, 2003.

PERES JUNIOR, J. et al. Perfil parasitológico e microbiológico de alfaces comercializadas em restaurantes self-service de Gurupi-TO. Revista Científica do ITPAC, v. 5 , p. 1-8, 2012.

ROBERTSON, L.J.; GJERDE, B. Ocurrence of parasites on fruits and vegetables in Norway. Journal of Food Protection, v.64, p.1793-1798, 2001.

SILVA, C. G. M.; ANDRADE, S. A. C.; STAMFORD, T. L. M. Occurrence of Cryptosporidium spp. and others parasites in vegetables consumed in natura, Recife, Brazil. Ciência e Saúde Coletiva, v. 10, p. 63-69, 2005.

SOARES, B.; CANTOS, G. A. Parasitological quality and hygienic-sanitary conditions of vegetables sold in the city of Florianópolis, Santa Catarina, Brazil. Revista Brasileira de Epidemiologia, v. 8, p. 377-384, 2005.

TEFERA, T. T. et al. Parasitic Contamination of Fruits 
and Vegetables Collected from Selected Local Markets of Jimma Town, Southwest Ethiopia. International Scholarly Research Notices, v. 2014, p. 1-7, 2014.

YUSOF, A. M. et al. Occurrence of Intestinal Parasitic Contamination in Select Consumed Local Raw Vegetables and Fruits in Kuantan, Pahang. Tropical Life Sciences Research, v. 28, p. 23-32, 2017.

ZUMAQUERO-RÍOS, J. L. et al. Fascioliasis and Intestinal Parasitoses Affecting Schoolchildren in Atlixco, Puebla State, Mexico: Epidemiology and Treatment with Nitazoxanide. PLOS Neglected Tropical Diseases, v. 7, p. 1-16, 2013.

Recebido em: 27.02.2018 Aceito em: 08.06.2018 